\title{
Induction of Bim limits cytokine-mediated prolonged survival of neutrophils
}

\author{
N Andina ${ }^{1}$, S Conus ${ }^{1}$, EM Schneider ${ }^{2}$, MF Fey ${ }^{3}$ and HU Simon ${ }^{*, 1}$
}

\begin{abstract}
Under inflammatory conditions, neutrophil apoptosis is delayed due to survival-factor exposure, a mechanism that prevents the resolution of inflammation. One important proinflammatory cytokine involved in the regulation of neutrophil survival/activation is granulocyte-macrophage colony-stimulating factor (GM-CSF). Although GM-CSF mediates antiapoptotic effects in neutrophils, it does not prevent apoptosis, and the survival effect is both time dependent and limited. Here, we identified the proapoptotic Bcl-2 family member Bim as an important lifespan limiting molecule in neutrophils, particularly under conditions of survival factor exposure. Strikingly, GM-CSF induced Bim expression in both human and mouse neutrophils that was blocked by pharmacological inhibition of phosphatidylinositol-3 kinase (PI3K). Increased Bim expression was also seen in human immature bone marrow neutrophils as well as in blood neutrophils from septic shock patients; both cell populations are known to be exposed to GM-CSF under in vivo conditions. The functional role of Bim was investigated using Bim-deficient mouse neutrophils in the presence and absence of the survival cytokines interleukin (IL)-3 and GM-CSF. Lack of Bim expression resulted in a much higher efficacy of the survival cytokines to block neutrophil apoptosis. Taken together, these data demonstrate a functional role for Bim in the regulation of neutrophil apoptosis and suggest that GM-CSF and other neutrophil hematopoietins initiate a proapoptotic counterregulation that involves upregulation of Bim.
\end{abstract}

Cell Death and Differentiation (2009) 16, 1248-1255; doi:10.1038/cdd.2009.50; published online 1 May 2009

Neutrophils are important effector cells of the immune system. They are constantly produced in large numbers in the bone marrow. After maturation they enter the blood circulation and usually die within a short period of time due to apoptosis. ${ }^{1,2}$ Thus, neutrophil numbers are controlled by the rates of both differentiation and apoptosis. During bacterial and fungal infections, it is well known that neutrophil numbers increase due to a higher production of these cells. However, neutrophil apoptosis is also delayed under these conditions, largely due to the same cytokines, which drive their differentiation, particularly granulocyte colony-stimulating factor (G-CSF) and granulocyte-macrophage colony-stimulating factor (GM-CSF). ${ }^{3,4}$ Decreased rates of neutrophil apoptosis maintain inflammation and prevent the resolution of inflammatory responses in vivo. ${ }^{5,6}$ During the resolution of inflammation, increased neutrophil apoptosis occurs and apoptotic neutrophils are phagocytosed by other cells. ${ }^{7}$

Though it is clear that apoptosis contributes to the regulation of neutrophil numbers under both physiologic and pathologic conditions, it remains unclear how the process of lifespan prolongation of mature neutrophils by survival cytokines is limited. For instance, what mechanisms control the expansion of neutrophils during inflammatory processes? Why does neutrophil apoptosis, although delayed, still occur? What limits the lifespan of neutrophils exposed to survival cytokines? Clearly, these questions are of fundamental importance to our understanding of how inflammation is regulated and address obvious therapeutic implications.

Apoptosis of neutrophils under conditions of cytokine withdrawal occurs via the mitochondria-dependent death pathway, which is known to be regulated by members of the Bcl-2 family. ${ }^{1}$ These proteins share one or more Bcl-2 homology $(\mathrm{BH})$ domains and can be divided into prosurvival proteins (such as A1, Bcl-2, Bcl- $\mathrm{x}_{\mathrm{L}}, \mathrm{Mcl}-1$ ), proapoptotic Bax/ Bak-like proteins that contain more than one $\mathrm{BH}$-domain, and proapoptotic BH3-only proteins (such as Bad, Bim, Bid, Noxa, Puma). ${ }^{8}$ It is now widely accepted that the balance between proapoptotic and prosurvival $\mathrm{Bcl}-2$-like relatives determines cell fate. ${ }^{8}$

Under steady-state conditions, neutrophils express high levels of proapoptotic but only small amounts of antiapoptotic Bcl-2 family members. ${ }^{3,9,10}$ Survival cytokines appear to mediate their antiapoptotic effects, at least partially, by increasing $\mathrm{Mcl}-1,{ }^{10-12} \mathrm{~A} 1,{ }^{10,13}$ and/or Bcl- $\mathrm{x}_{\mathrm{L}} \cdot{ }^{3,9,14}$ In contrast, mature neutrophils did not demonstrate detectable levels of Bcl-2 on GM-CSF or G-CSF stimulation. ${ }^{3,15}$ Moreover, the expression of proapoptotic proteins, such as Bad, Bax, Bak, or Bik, was unaffected under conditions of delayed neutrophil apoptosis. $^{10}$

The proapoptotic $\mathrm{BH} 3$-only protein Bim is thought to play a major role in promoting cell death following withdrawal of survival factors. For instance, it has been shown that Bim

\footnotetext{
${ }^{1}$ Institute of Pharmacology, University of Bern, Bern, Switzerland; ${ }^{2}$ Sektion Experimentelle Anästhesiologie, Universitätsklinikum Ulm, Ulm, Germany and ${ }^{3}$ Department of Medical Oncology, Inselspital, University of Bern, Bern, Switzerland

${ }^{*}$ Correspondence: HU Simon, Institute of Pharmacology, University of Bern, Friedbühlstrasse 49, CH-3010 Bern, Switzerland.

Tel: + 4131632 3281; Fax: + 4131632 4992; E-mail: hus@pki.unibe.ch

Keywords: Bim; cell death; GM-CSF; interleukin-3; neutrophils; sepsis

Abbreviations: IL, interleukin; Bim $\mathrm{EL}_{\mathrm{L}}$, Bim extra long; Bim, Bim long; Bim $\mathrm{S}_{\mathrm{S}}$ Bim short; DLC, dynein light chain; GAPDH, glyceraldehyde-3-phosphate dehydrogenase; Mcl-1, myeloid-cell leukemia sequence 1

Received 18.11.08; revised 13.3.09; accepted 30.3.09; Edited by RA Knight; published online 01.5.09
} 
is de novo synthesized in several hematopoietic cell lines, primary neurons, osteoclasts, and fibroblasts following withdrawal of survival factors. ${ }^{16-21}$ Moreover, $\mathrm{Bim}^{-1-} \mathrm{B}$ and T cells as well as granulocytes show relative resistance to cytokine deprivation. $^{21,22}$ Here, we report the surprising finding that some survival cytokines induce Bim gene expression in neutrophils. The induction of proapoptotic Bim by antiapoptotic cytokines seems to be of fundamental importance to limit neutrophil antiapoptosis, as Bim deficiency dramatically enhanced the lifespan of neutrophils in the presence of survival cytokines. We therefore provide evidence that survival cytokines not only initiate antiapoptotic and thereby proinflammatory molecular events in neutrophils, but also induce mechanisms required for inflammation limitation and resolution, respectively.

\section{Results}

Bim expression is reduced during terminal differentiation of neutrophils. We investigated Bim expression on purified immature bone marrow and mature blood neutrophil populations using immunoblot analysis. Both immature and mature neutrophils expressed all three known isoforms of Bim: Bim $\mathrm{EL}_{\mathrm{L}}$, Bim $\mathrm{L}_{\mathrm{L}}$ and Bim $\mathrm{Bim}_{\mathrm{EL}}$ was always higher expressed compared with $\mathrm{Bim}_{\mathrm{L}}$ and $\mathrm{Bim}_{\mathrm{S}}$ (Figure 1). The three isoforms of Bim were identified at the

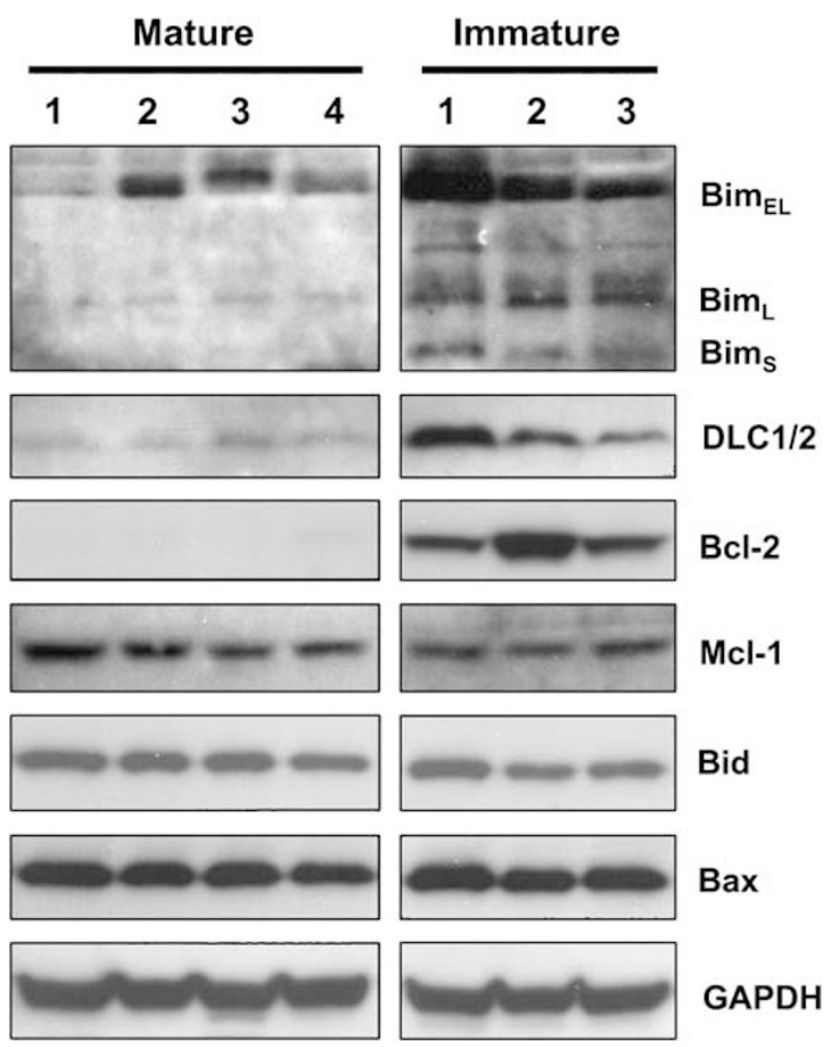

Figure 1 Expression of $\mathrm{Bcl}-2$ family members in freshly purified immature and mature human neutrophils as assessed by immunoblotting. DLC1/2 expression was also investigated. Filters were reprobed with anti-GAPDH mAb to ensure equal loading of the gels. For both immature and mature neutrophil populations, results from different donors are shown same molecular size as found in human embryonic kidney HEK-293 cells, which were used as a positive control (data not shown). ${ }^{23}$ Moreover, Bim expression was lower in mature neutrophils compared with immature neutrophils (Figure 1). We also analyzed the expression of dynein light chains (DLC) 1 and 2 that were described as being able to sequester Bim and Bmf, respectively. ${ }^{24,25}$ DLC1/2 was seen at much higher levels in immature neutrophils compared with mature neutrophils (Figure 1), a finding, which may explain, at least partially, why immature neutrophils tolerate high Bim levels without undergoing rapid apoptosis. ${ }^{26}$

In the course of these experiments, we also investigated the expression of other members of the Bcl-2 family in both immature and mature neutrophils. Bcl-2 expression was clearly detectable in immature but not in mature neutrophils (Figure 1). Similarly, Bcl- $x_{L}$ was seen in immature but not in mature neutrophils (data not shown). In contrast to Bcl-2 and $\mathrm{Bcl}-\mathrm{x}_{\mathrm{L}}, \mathrm{Mcl}-1$ was found equally expressed in immature and mature neutrophils, suggesting that it does not disappear during terminal differentiation. The proapoptotic members Bid and Bax were also expressed at similar levels in both immature and mature neutrophils.

Bim expression is inducible in neutrophils on GM-CSF and G-CSF stimulation. During maturation, neutrophils are exposed to high levels of cytokines in the bone marrow that regulate gene expression. Some of these cytokines, such as GM-CSF and G-CSF, are also elevated in bacterial infectious diseases., ${ }^{3,4}$ We therefore tested the hypothesis whether these cytokines are able to induce Bim in mature neutrophils. In initial experiments, we observed that Bim mRNA was 12.64-fold induced in neutrophils following $7 \mathrm{~h}$ GM-CSF stimulation using an oligonucleotide microarray assay, in which we screened the regulation of 12599 genes in these cells. $^{27}$ This finding was striking, because GM-CSF is known as a neutrophil survival factor. To validate these findings at the protein level, we performed immunoblotting experiments. Indeed, both GM-CSF and G-CSF also induced proapoptotic Bim protein expression in neutrophils (Figure 2a). Increased expression of all Bim isoforms was observed, although levels of $\mathrm{Bim}_{\mathrm{EL}}$ were particularly enhanced (Figure $2 \mathrm{~b}$ ). Moreover, this isoform exhibited an apparent higher molecular weight (Figure 2a), perhaps as a consequence of phosphorylation. ${ }^{21}$ Indeed, treatment of protein extracts with $\lambda$-phosphatase demonstrated that the modification of $\mathrm{Bim}_{\mathrm{EL}}$ was due to a change in phosphorylation (Figure 2c). In addition, we noticed a small increase of Bim expression in cultured neutrophils derived from most normal donors in the absence of survival factors (Figure 2a,b). Time-course experiments revealed that Bim levels were increased within $8 \mathrm{~h}$ in mature neutrophils on stimulation with GM-CSF, peaked at $12 \mathrm{~h}$, and higher Bim levels were maintained up to $24 \mathrm{~h}$ (Figure $2 \mathrm{~d}$ ).

In the course of these experiments, we obtained the following additional information: GM-CSF and G-CSF maintained or slightly increased Mcl-1 levels for a limited time period that, however, rapidly declined in the absence of cytokine support (Figure 2d). Bid levels also declined more rapidly in the absence of survival cytokines (Figure 2d). In contrast, Bax levels were not affected by GM-CSF and G-CSF stimulation (Figure 2d). In addition, we did not observe 


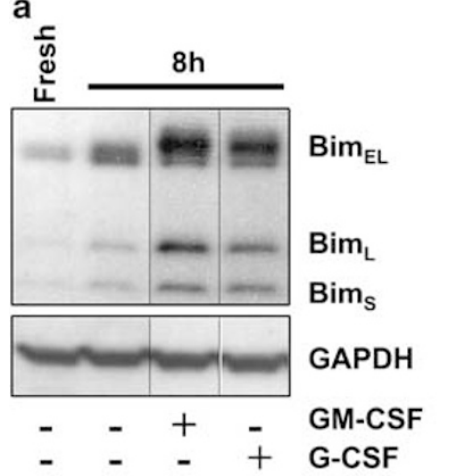

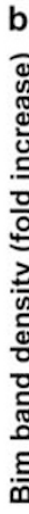

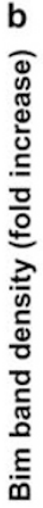

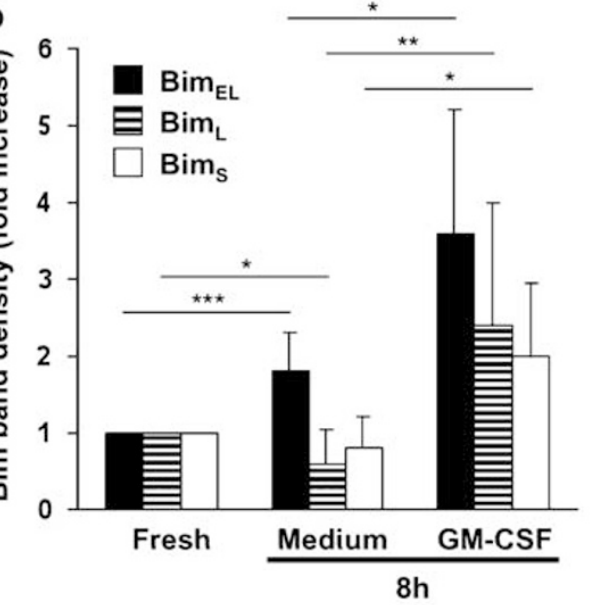

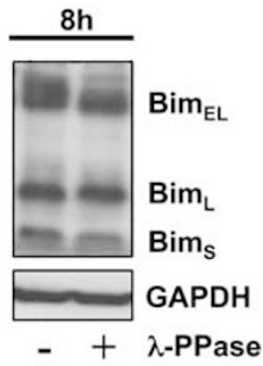

d

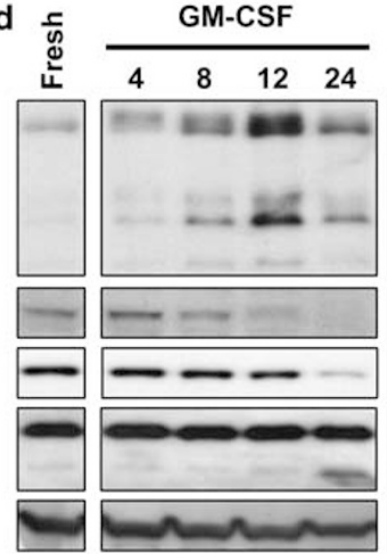

Medium

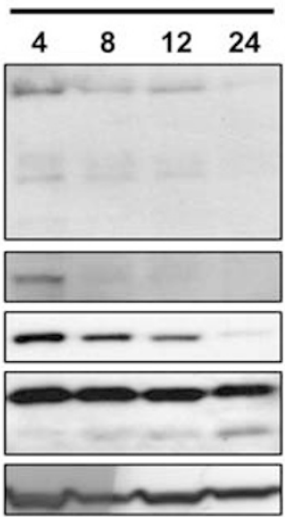

Time (h)
Bim $_{E L}$
Bim $_{L}$
Bim $_{S}$
Mcl-1
Bid
Bax
tBax
GAPDH

e

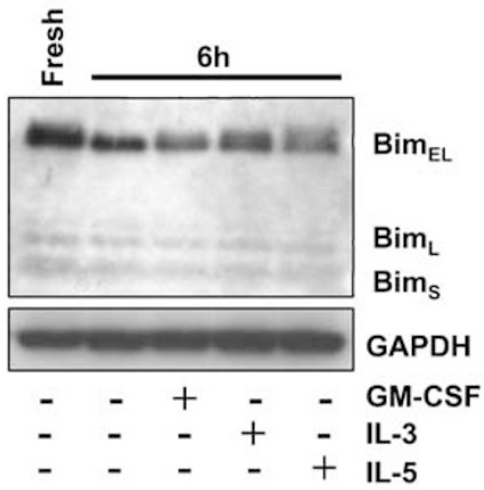

Figure 2 Bim levels are increased in mature neutrophils exposed to survival factors in vitro as assessed by immunoblotting. (a) Expression of Bim in freshly isolated neutrophils and in 8-h neutrophil cultures in the presence and absence of GM-CSF and G-CSF, respectively. The results are representative of eight independent experiments. (b) Quantitative analysis of the Bim experiments shown in (a) performed by densitometry. Values are mean \pm S.D. of eight independent experiments. ${ }^{*} P<0.05$; ${ }^{* *} P<0.01$; ${ }^{* * \star} P<0.001$. (c) Posttranslational modification of Bim. Neutrophils were cultured in the presence of GM-CSF for $8 \mathrm{~h}$. Protein extracts were prepared in the absence of phosphatase inhibitors and left untreated or treated with $\lambda$-phosphatase ( $\lambda$-PPase) before analyzing Bim. The results are representative of three independent experiments. (d) Time-dependent expression of Bim and other Bcl-2 family members in neutrophils following GM-CSF stimulation. The results are representative of three independent experiments. (e) Expression of Bim in freshly isolated eosinophils and in 6-h eosinophil cultures in the presence and absence of GM-CSF, IL-3, and IL-5. The results are representative of three independent experiments

increased $\mathrm{Bcl}-2, \mathrm{Bcl}-\mathrm{x}_{\mathrm{L}}$, and $\mathrm{DLC1/2}$ levels under these conditions in neutrophils (data not shown). We also tested whether eosinophils exhibit higher Bim levels after survival factor stimulation. In contrast to neutrophils, Bim levels were the same in the presence and absence of IL-3, IL-5, and GM-CSF in 6-h cultured eosinophils (Figure 2e). We also did not obtain evidence for cytokine-mediated increases in Bim expression in eosinophils at later time points (data not shown).

GM-CSF-mediated increase in Bim expression is the consequence of transcriptional activation of the Bim gene and involves the PI3K pathway. We treated neutrophils with actinomycin $D$ and cycloheximide to block transcription and translation, respectively. Pharmacological inhibition of both transcription and translation blocked GMCSF-mediated increases in Bim expression (Figure 3a), suggesting that increased Bim levels are likely due to transcriptional activation of the Bim gene. Indeed, GM-CSF rapidly induced Bim mRNA expression as assessed by a quantitative real-time PCR technique (Figure 3b). These findings confirmed our initial observation using oligonucleotide microarrays that suggested increased Bim mRNA levels in neutrophils exposed to GM-CSF.

To test whether activation of the Bim gene involves the activation of PI3K and/or mitogen-activated protein kinase (MAPK) pathways, mature human neutrophils were preincubated with different concentrations of defined kinase inhibitors. LY294002, an inhibitor of PI3K, completely prevented GM-CSF-mediated increases in Bim expression and survival (Figure 4a). A time-course experiment revealed that the presence of LY294002 did not allow Bim increases at any time point (Figure 4b). The extracellular-signal regulated kinase (ERK) inhibitor PD098059 partially blocked survival and increased the expression of all splice variants of Bim in the presence of GM-CSF, but had no effect on the mobility shift of Bim $_{\mathrm{EL}}$ (Figure 4c). On the other hand, the p38 MAPK inhibitor SB203580 and the Jun kinase inhibitor (JNK) inhibitor SP600125 had no influence on Bim levels in 

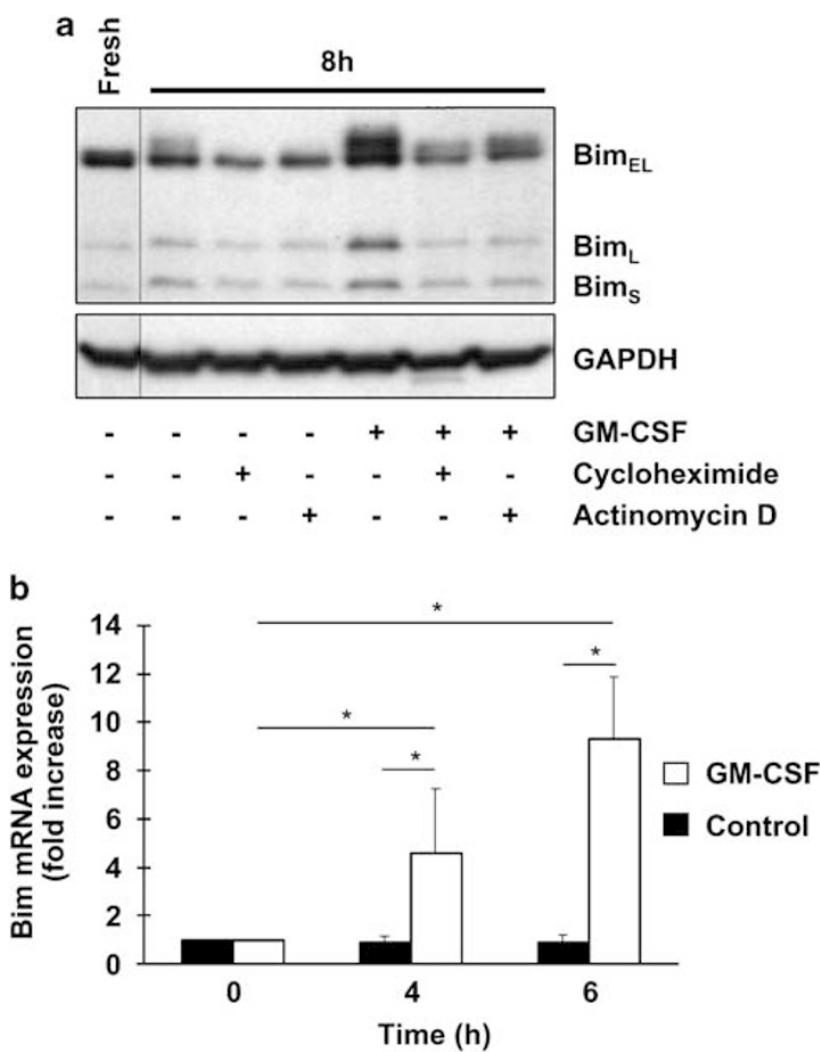

Figure 3 Increased Bim gene expression in neutrophils following GM-CSF stimulation in vitro. (a) Immunoblotting: Expression of Bim in 8-h GM-CSFstimulated neutrophil cultures in the presence and absence of cycloheximide and actinomycin $D$, respectively. Two independent experiments with same results were performed. (b) Real-time PCR: Time-dependent expression of Bim mRNA results in GM-CSF-stimulated neutrophils. Results are expressed as fold increase compared with freshly isolated cells. Values are mean \pm S.D. of three independent experiments. ${ }^{*} P<0.05$

GM-CSF-stimulated neutrophils (Figure 4d). These data suggest that the PI3K pathway is required for the induction of the Bim gene, and that this pathway is partially blocked by ERK-mediated signaling events.

Increased Bim expression in sepsis neutrophils. We hypothesized at this point that Bim levels might be higher in patients suffering from bacterial infections. Indeed, neutrophils derived from the blood of septic shock patients demonstrated evidence for increased Bim expression in spite of increased viability compared with blood neutrophils from healthy control individuals (Figure 5). Although it cannot be excluded that this might be partially due to the occurrence of immature neutrophils in blood of septic shock patients (we observed strong $\mathrm{Bcl}-2$ expression in one of three patients), the data point to the possibility that mature neutrophils are triggered to express higher levels of Bim under inflammatory conditions in vivo. In contrast to Bim, Bax and Bid levels were not affected (Figure 5), suggesting that both terminal differentiation (Figure 1) and the inflammatory environment in septic shock patients does not influence Bax and Bid levels in neutrophils.
Bim limits prolonged cytokine-mediated survival of neutrophils. To address Bim functions in neutrophils, we switched to an in vitro mouse system using Bim-deficient animals. In initial experiments, normal mature bone marrow neutrophils from $\mathrm{Bim}^{+/+}$and $\mathrm{Bim}^{+/-}$mice were isolated, and then cultured in the presence and absence of GM-CSF and IL-3, respectively, for $8 \mathrm{~h}$. As expected, Bim levels were lower in $\mathrm{Bim}^{+/-}$compared with $\mathrm{Bim}^{+/+}$mice (Figure 6a). Neutrophils from both mouse strains, however, demonstrated increased Bim levels after stimulation with GM-CSF and IL-3, respectively (Figure 6a), suggesting that mouse mature neutrophils represent a suitable model to study the role of Bim on the regulation of neutrophil death both in the presence and absence of survival factors.

We, therefore, purified neutrophils from $\mathrm{Bim}^{+/+}, \mathrm{Bim}^{+/-}$, and $\mathrm{Bim}^{-1-}$ mice. Mature neutrophils were isolated from both bone marrow and blood, and then cultured for the indicated times. Spontaneous neutrophil death was delayed in $\mathrm{Bim}^{-1-}$ compared with $\mathrm{Bim}^{+/+}$mice, confirming previously published work. ${ }^{22}$ In addition, we obtained evidence for a clear genedosage effect as neutrophils from $\mathrm{Bim}^{+/-}$mice demonstrated intermediate values in this system (Figure 6b, left panels). The differences between wild-type and Bim-deficient neutrophils were even more pronounced under conditions of survival factor exposure. Bone marrow $\mathrm{Bim}^{-1-}$ neutrophils exposed to IL-3 did not die in the time period between 24 and $96 \mathrm{~h}$, whereas wild-type neutrophils demonstrated some cell death under these conditions (Figure 6b, upper right panel). Similarly, GM-CSF delayed much stronger neutrophil death in $\mathrm{Bim}^{-1-}$ compared with wild-type bone marrow neutrophils (Figure 6b, lower right panel). Blood neutrophils behaved similar as bone marrow neutrophils: IL-3 or GM-CSF and Bim-deficiency synergistically prolonged neutrophil survival (data not shown).

\section{Discussion}

Survival factor deprivation is thought to induce the Bim gene, resulting in cell death, and, readdition of the survival factors after withdrawal is believed to reduce Bim expression associated with increased cell survival. ${ }^{16-21}$ In this paper, we demonstrate that neutrophils, which represent one of the most frequent cell types in multicellular organisms, do not follow this dogma. In contrast to other cells, we show that survival cytokines (GM-CSF and G-CSF) induce Bim gene expression in neutrophils. It is possible that cytokine-mediated Bim upregulation is specific for neutrophils, because we did not observe elevated Bim levels in eosinophils on IL-3, IL-5, and GM-CSF stimulation. In addition, dendritic cells have been shown to downregulate Bim in the presence of GM-CSF. ${ }^{28}$

Survival factor induced upregulation of proapoptotic $\mathrm{Bcl}-2$ family members in neutrophils appears to be specific for Bim, because we did not observe changes in the expression of Bax and Bid. Moreover, Bak, Bad, and Bik expressions were also reported to be unaffected in neutrophils on GM-CSF treatment. ${ }^{10}$ We performed some initial experiments using pharmacological inhibitors to gain some insights into the regulation of the Bim gene in neutrophils. Surprisingly, we obtained evidence that PI3K is a crucial element in this 
a

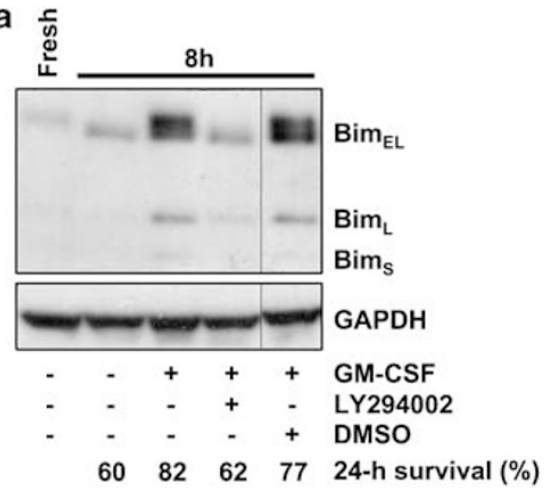

c

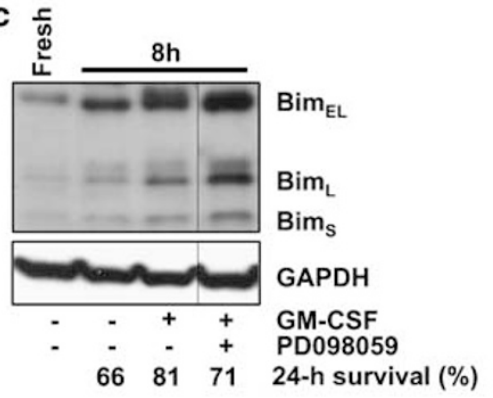

b

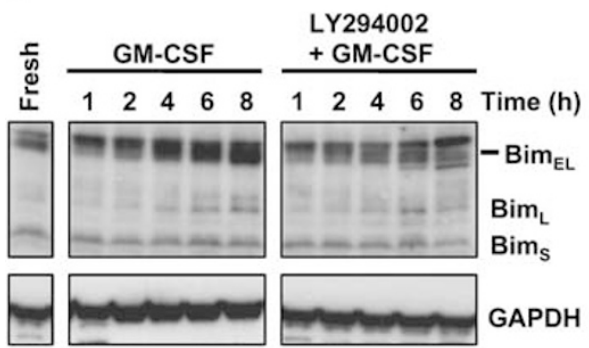

d

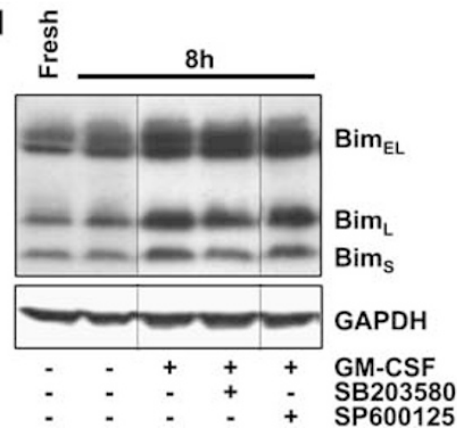

Figure 4 Increased Bim expression in neutrophils following GM-CSF stimulation in vitro is blocked by pharmacological inhibition of PI3K as assessed by immunoblotting (a) Expression of Bim in 8-h GM-CSF-stimulated neutrophil cultures in the presence and absence of LY294002. 24-h neutrophil survival of the analyzed cells was measured within the same experiments (bottom). (b) Time-dependent expression of Bim in GM-CSF-stimulated neutrophil cultures in the presence and absence of LY294002. Note that the protein above Bim $\mathrm{EL}_{\mathrm{L}}$ is nonspecifically detected by the anti-Bim antibody. (c) Expression of Bim in 8-h GM-CSF-stimulated neutrophil cultures in the presence and absence of PD098059. 24-h neutrophil survival of the analyzed cells was measured within the same experiments (bottom). (d) Expression of Bim in 8-h GM-CSF-stimulated neutrophil cultures in the presence and absence of SB203580 and SP600125, respectively. Results in all panels are representative of at least three independent experiments

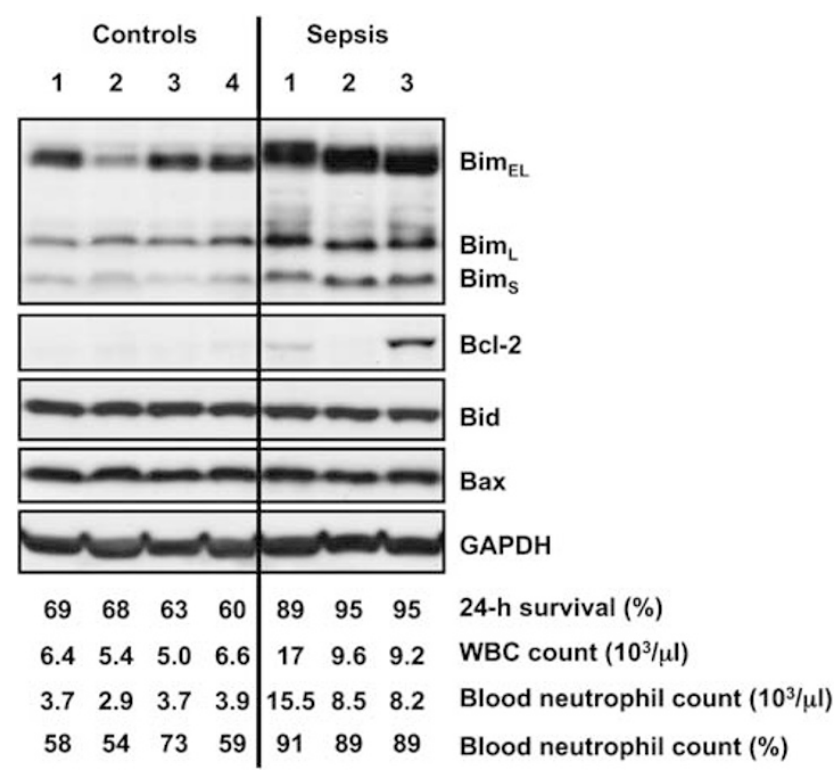

Figure 5 Increased Bim levels in freshly isolated neutrophils derived from patients suffering from septic shock as assessed by immunoblotting. Bcl-2, Bid, and Bax were also analyzed. Patients with septic shock exhibited several abnormalities regarding neutrophil numbers and 24-h neutrophil survival (bottom: Results correspond to the expression levels shown in the immunoblots). Results from different donors are shown pathway. In other cells, it has been reported that $\mathrm{PI} 3 \mathrm{~K}$ is rather involved in the downregulation of Bim expression ${ }^{17,29-34}$ or in Bim $_{E L}$ phosphorylation resulting in reduced Bim activity. ${ }^{21,35}$

That Bim levels do not necessarily correlate with cell survival has been reported in other experimental cell death systems. For instance, toll-like receptor (TLR) stimulation induced Bim in macrophages in the absence of marked apoptosis. ${ }^{36}$ Moreover, TLR stimulation increased survival of dendritic cells and concomitantly induced $\mathrm{Bcl}-\mathrm{x}_{\mathrm{L}}, \mathrm{Mcl}-1$, and Bim. ${ }^{28}$ FcåRl activation increased mast cell survival and, at the same time, levels of both $\mathrm{BCl}-\mathrm{x}_{\mathrm{L}}$ and Bim. ${ }^{37} \mathrm{LPS}, \mathrm{IL}-15$, $\mathrm{IL}-1$, and IL-7 increased T-cell survival and induced Bim, but did not affect the expression of the prosurvival proteins Bcl-2, $\mathrm{BCl}-\mathrm{x}_{\mathrm{L}}$, and $\mathrm{Mcl}-1{ }^{38}$ It has also been reported that the expression of $\mathrm{Mcl}-1, \mathrm{~A} 1$, and Bim increases in $24 \mathrm{~h}$ mouse neutrophil cultures in the presence of LPS compared with cells cultured in medium alone. ${ }^{38}$ However, as the authors of the latter study did not include freshly isolated mouse neutrophils in their experimental settings, it remained unclear whether LPS induced the Bim gene or whether it just maintained Bim levels for a longer period of time. Most of these studies show that increased Bim levels occur in association with increases in the expression of members of the $\mathrm{Bcl}-2$ family with prosurvival activities. This could explain why these cells survive despite of elevated Bim levels. In neutrophils, the most likely candidate that might fulfill this function is Mcl-1. ${ }^{10-12}$ 

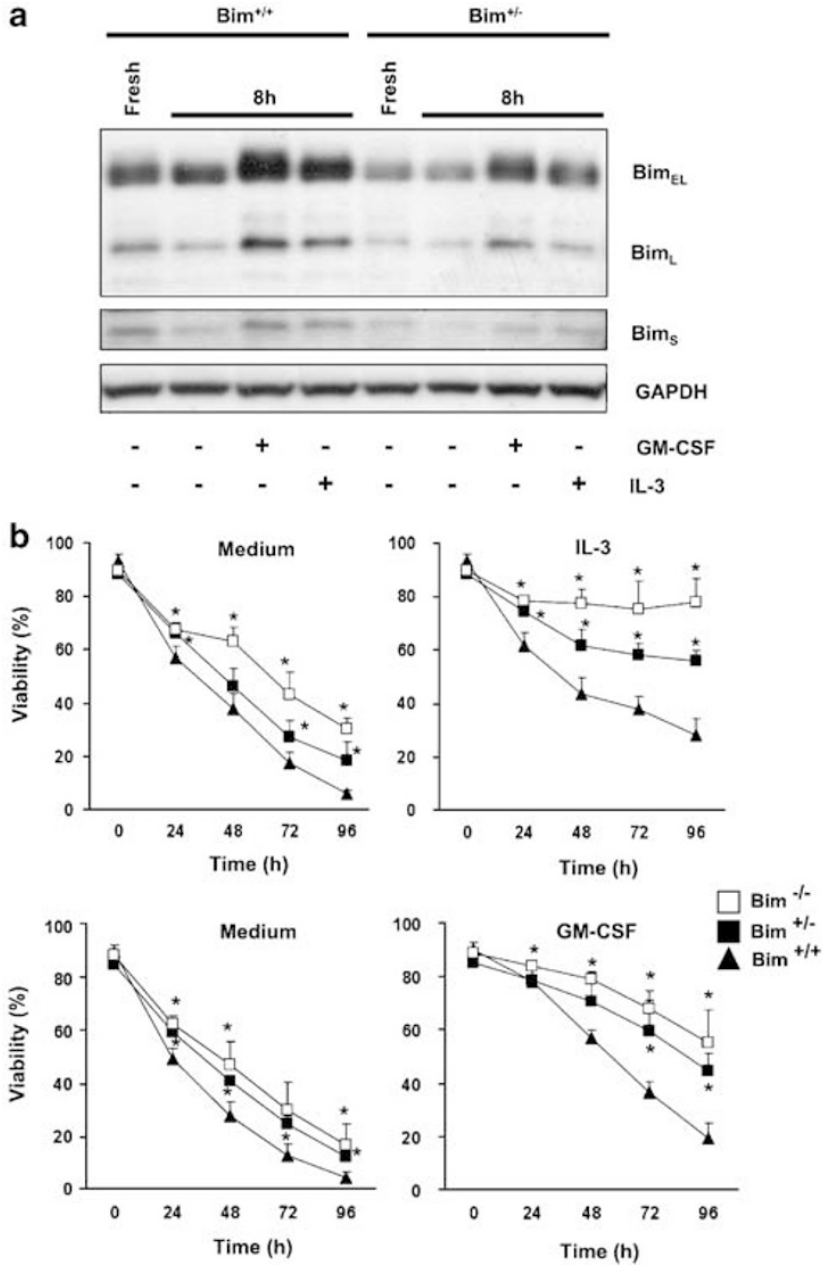

Figure 6 Death of mature mouse bone marrow neutrophils in the presence of survival factors is largely dependent on Bim. (a) Immunoblotting: Expression of Bim in freshly isolated mouse neutrophils and in 8-h mouse neutrophil cultures in the presence and absence of GM-CSF and IL-3, respectively. Neutrophils were isolated from $\mathrm{Bim}^{+1+}$ and $\mathrm{Bim}^{+1-}$ mice. For the detection of $\mathrm{Bim}_{\mathrm{S}}$, the film was overexposed. (b) Viability assay: Mature mouse neutrophils derived from bone marrow were cultured in the absence or presence of IL-3 (upper panels: $\mathrm{Bim}^{+1+}$ $(n=4), \mathrm{Bim}^{+l-}(n=6)$, and $\left.\mathrm{Bim}^{-1-}(n=5)\right)$ or GM-CSF (lower panels: Bim ${ }^{+1+}$ $(n=3), \mathrm{Bim}^{+-}(n=6)$, and $\left.\mathrm{Bim}^{-1-}(n=5)\right)$ for the indicated time periods and then analyzed. Values are mean \pm S.D. Same results were obtained using blood neutrophils. ${ }^{*} P<0.05$

Besides proapoptotic Bcl-2 family members, other mechanisms may also contribute to the regulation of Bim activity. For instance, it has been shown that both $\operatorname{Bim}_{E L}$ and $\operatorname{Bim}_{L}$ can be sequestered by the microtubular dynein-motor complex through binding to DLC1. ${ }^{24}$ On the other hand, it was found that most of the cellular $\operatorname{Bim}_{\mathrm{EL}}$ and $\mathrm{Bim}_{\mathrm{L}}$ is localized at the mitochondria and is hardly associated to microtubules in primary $T$ and $B$ cells. ${ }^{39,40}$ We found increased levels of DLC1/2 in immature compared with mature neutrophils. In contrast to Bim, DCL1/2 were not inducible in mature neutrophils stimulated with GM-CSF. This might explain why Bim is functionally active in mature neutrophils under these conditions.

In this study, we observed a marked mobility shift of the extra-long form of Bim following survival factor stimulation in neutrophils. However, this shift was not complete, leaving a considerable amount of unphosphorylated $\mathrm{Bim}_{\mathrm{EL}}$. Furthermore, although phosphorylation of Bim may suggest the partial inactivation of the extra-long form, it should be noted that the long and short forms did not seem to be affected. The potential GM-CSF-mediated Bim $\mathrm{EL}_{\mathrm{L}}$ phosphorylation in neutrophils most likely involves PI3K, as the mobility shift of Bim $_{E L}$ was not seen in the presence of LY294002. Phosphorylation of $\mathrm{Bim}_{\mathrm{EL}}$ has been reported to be $\mathrm{PI} 3 \mathrm{~K},{ }^{35} \mathrm{ERK},{ }^{21}$ or $\mathrm{JNK}^{21}$ dependent. Most studies concluded that phosphorylated Bim $\mathrm{EL}_{\mathrm{L}}$ would result in reduced proapoptotic activity, but the opposite has also been observed. ${ }^{21}$ In conclusion, the effects of Bim $\mathrm{EL}$ phosphorylation on Bim activity and neutrophil survival remain to be investigated. Nevertheless, the results of this study demonstrate a proapoptotic role of Bim in neutrophils both in the presence and absence of survival cytokines.

The precise investigation of molecular mechanisms in neutrophils has been difficult, because the cells are fragile and undergo rapid spontaneous apoptosis. Plasmid transfection appeared to be impossible in neutrophils, ${ }^{41}$ limiting the common approaches to study apoptotic pathways in these cells. Therefore, to investigate a possible function of Bim, we assessed cell death of neutrophils derived from wild-type and Bim-deficient mice. We demonstrate in this study that the presence of Bim largely reduces the lifespan of these cells, particularly when exposed to survival cytokines. Moreover, Bim deficiency has been demonstrated to promote neutrophil accumulation in vivo. ${ }^{16}$ Therefore, increased levels of Bim in neutrophils under inflammatory conditions may represent an important mechanism, which limits inflammatory responses.

Bim is not the first identified molecule that counterregulates inflammatory responses. For instance, cytokines also induce members of the suppressors or cytokine signaling (SOCS) family that antagonize subsequent cytokine signaling in neutrophils. ${ }^{42}$ Downregulation of cytokine receptors may also lead to cytokine unresponsiveness of these cells. ${ }^{43}$ Moreover, neutrophils have been described to switch their lipid mediator production from leukotrienes to antiinflammatory lipoxins ${ }^{44}$ and to generate IL-10. ${ }^{45}$ Taken together, the results of this study suggest that the induction of the Bim gene represents one important molecular event occurring in neutrophils early in inflammation that, in concert with other counterregulatory mechanisms, contributes to the limitation of inflammatory responses and/or to their resolutions.

\section{Materials and Methods}

Reagents. Recombinant cytokines were purchased as follows: human GM-CSF was from Novartis Pharma GmbH (Nürnberg, Germany), human G-CSF was from Aventis Pharma AG (Zürich, Switzerland), human IL-5 and mouse GM-CSF were from R\&D Systems Europe Ltd (Abingdon, UK), and mouse IL-3 was from BD Biosciences (Basel, Switzerland). The pharmacological inhibitors actinomycin D, cycloheximide, LY294002, SB203580, and SP600125 were from CalbiochemNovabiochem Corp. (La Jolla, CA, USA). PD98059 was from Alexis (Lausen, Switzerland). Antibodies were obtained as follows: anti-human Bax was from BD Biosciences, anti-human Bcl-2 mAb was from Dako (Baar, Switzerland), anti-human Bid was from R\&D Systems, anti-Bim Abs were from Stressgen Bioreagents (Victoria, BC, Canada) and Sigma-Aldrich (Buchs, Switzerland), anti$\mathrm{DLC} 1 / 2 \mathrm{mAb}$ (this mAb detects both DLC1and DLC2) was from Alexis, anti-Mcl$1 \mathrm{mAb}$ was from Calbiochem-Novabiochem, and anti-GAPDH mAb was from Chemicon International Inc. (Juro, Lucerne, Switzerland). Horseradish peroxidase 
(HRP)-conjugated secondary Abs were from Amersham Bioscience Europe $\mathrm{GmbH}$ (Freiburg, Germany). Recombinant $\lambda$-phosphatase was obtained from New England Biolabs Inc. (Ipswich, MA, USA).

Cells. Mature blood neutrophils were isolated from peripheral blood of healthy donors and patients suffering from septic shock by Ficoll-Hypaque centrifugation. ${ }^{6,46}$ Briefly, peripheral blood mononuclear cells were separated by centrifugation on Ficoll-Hypaque (Seromed-Fakola AG, Basel, Switzerland). The lower phase, mainly granulocytes and erythrocytes, was treated with erythrocyte lysis solution ( $155 \mathrm{mmol} / / \mathrm{NH}_{4} \mathrm{Cl}, 10 \mathrm{mmol}^{\prime} \mathrm{KHCO}_{3}$, and $0.1 \mathrm{mmol} / \mathrm{IEDTA}$, pH 7.3). The resulting cell populations contained greater than $95 \%$ mature neutrophils as assessed by staining with Diff-Quik (Medion $\mathrm{GmbH}$, Düdingen, Switzerland) and light microscopy analysis.

Immature neutrophils were isolated from bone marrow aspirates with normal cellular morphology and distribution as described previously. ${ }^{26,47}$ Briefly, following centrifugation on a two-step discontinuous percoll density gradient, cells were negatively isolated using anti-CD7 and anti-CD36 mAbs to eliminate contaminating lymphoid and erythroid precursors. The resulting cell population contained $>97 \%$ cells of the neutrophil lineage as determined by myeloperoxidase staining, flow cytometric analysis of lineage-associated surface proteins, ${ }^{26}$ as well as with DiffQuik and light microscopy. The proportion of mature neutrophils was less than $5 \%$.

Peripheral blood eosinophils from control individuals were purified as described previously. ${ }^{48,49}$ The resulting cell populations contained more than $99 \%$ eosinophils as controlled by staining with Diff-Quik and light microscopy.

All individuals who donated blood or bone marrow aspirates signed a written informed consent agreement in accordance with the Declaration of Helsinki. The study was approved by the local medical ethics committee (the Kantonale Ethikkommission Bern).

Mature neutrophils were also isolated from the bone marrow and blood of $\mathrm{Bim}^{+1+}, \mathrm{Bim}^{+l-}$, and $\mathrm{Bim}^{-1-}$ mice (B6.129S1-Bcl2l11tm1.1Ast/J; Jackson Laboratory, Bar Habor, Maine, USA; kindly provided by Dr. Christoph Müller, Bern, Switzerland) as previously described. ${ }^{6}$ The purity of the resulting mouse neutrophil populations was $>90 \%$ (bone marrow) and $>95 \%$ (blood), respectively, as assessed by staining with Diff-Quick and light microscopy analysis. For all experiments, mice with a C57BL/6J background that were 5-8 weeks old were used. Mice were maintained under pathogen-free conditions. All animal experiments were reviewed and approved by the Animal Experimentation Review Board of the State of Bern.

Cell cultures. Human blood neutrophils and eosinophils, as well as mouse bone marrow neutrophils were cultured at $1 \times 10^{6} / \mathrm{ml}$. Mouse blood neutrophils were used at $25 \times 10^{4} / \mathrm{ml}$. Cells were cultures in complete culture medium (RPMI 1640 containing $10 \%$ fetal calf serum) in the presence or absence of GM-CSF (human: $10 \mathrm{ng} / \mathrm{ml}$; mouse: $0.1 \mathrm{ng} / \mathrm{ml}$ ), G-CSF $(25 \mathrm{ng} / \mathrm{ml})$, IL-3 (human: $10 \mathrm{ng} / \mathrm{ml}$; mouse: $1 \mu \mathrm{g} / \mathrm{ml})$, and IL-5 $(25 \mathrm{ng} / \mathrm{ml})$ for the indicated time periods. Neutrophils were also treated with actinomycin D $(10 \mu \mathrm{g} / \mathrm{ml})$, cycloheximide $(50 \mu \mathrm{g} / \mathrm{ml})$, LY294002 $(25 \mu \mathrm{M})$, SB203580 $(10 \mu \mathrm{M})$, SP600125 $(10 \mu \mathrm{M})$, and PD98059 $(50 \mu \mathrm{M})$. The inhibitors were added $30 \mathrm{~min}$ before cytokine stimulation.

Immunoblotting. Cultured cells were washed with phosphate-buffered saline (PBS) supplemented with a protease inhibitor cocktail (Sigma-Aldrich) and lysed with RIPA buffer ( $50 \mathrm{mM}$ Tris- $\mathrm{HCl}(\mathrm{pH} 7.4), 150 \mathrm{mM} \mathrm{NaCl}, 0.25 \%$ sodium deoxycholate, $1 \%$ Nonidet P- $40,1 \mathrm{mM}$ EGTA) supplemented with protease inhibitor cocktail with frequent vortexing on ice for $15 \mathrm{~min}$. To analyze possible Bim phosphorylation, extracts were left untreated or treated with $\lambda$-phosphatase for $30 \mathrm{~min}$ at $30^{\circ} \mathrm{C} .^{50}$ After a 10 -min centrifugation to remove insoluble particles, equal amounts of the cell lysates were loaded on gels (NuPage; Invitrogen). Separated proteins were electrotransferred onto polyvinylidene difluoride (PVDF) membranes (Immobilon-P; Millipore, Bedford, MA, USA). The filters were incubated overnight at $4{ }^{\circ} \mathrm{C}$ in TBS $/ 0.1 \%$ Tween-20/5\% nonfat dry milk with anti-Bax, anti-Bcl-2, anti-Bid, anti-Bim, anti-DLC1/2, and anti-Mcl-1 Abs (all at 1/1000). For loading controls, stripped filters were incubated with anti-GAPDH mAb (1/10000). Filters were washed in TBS/0.1\% Tween-20/5\% nonfat dry milk for $30 \mathrm{~min}$ at room temperature and incubated with the appropriate HRP-conjugated secondary Ab (Amersham) in TBS $/ 0.1 \%$ Tween-20/5\% nonfat dry milk for $1 \mathrm{~h}$. Filters were developed by an enhanced chemiluminescence technique (ECL kit; Amersham) according to the manufacturer's instructions.

Determination of cell death. Cell death was assessed by uptake of $1 \mu \mathrm{M}$ ethidium bromide and flow cytometric analysis (FACS Calibur; BD Biosciences). ${ }^{6,46,47}$
Quantitative real-time PCR (TaqMan). Total RNA $(1 \mu \mathrm{g})$ isolated with Trizol reagent (Invitrogen) was used for reverse transcriptase PCR (iScript select cDNA synthesis kit; Bio-Rad, Basel, Switzerland) using random primer for amplification. After CDNA synthesis, $1 \mu \mathrm{l}$ of PCR product was used for further analysis. The real-time PCR reaction was carried out in PCR plates from ABgen (Thermo-Fast 96 Detection Plate; Axonlab, Baden, Switzerland). Probes and primers for human Bim (hs00197982_m1) and 18S were obtained from Applied Biosystems (Warrington, UK) and the reporter dyes chosen were FAM and VIC, respectively. The PCR buffer used in the experiments was from ABgene (Absolute QPCR ROX Mix; Axonlab). The run was performed on the iQ5 Multicolor Real-Time PCR detection system from Bio-Rad. The cycling conditions were as follows: $95^{\circ} \mathrm{C}$ for $15 \mathrm{~min}$ ( 1 cycle), $95^{\circ} \mathrm{C}$ for $15 \mathrm{~s}$, and $60^{\circ} \mathrm{C}$ for $1 \mathrm{~min}$ ( 40 cycles). The Bio-Rad iQ5 2.0 Standard edition optical system software was used to analyze real time and endpoint fluorescence.

Statistical analysis. Statistical analysis was performed with the Mann-Whitney $U$ test. If mean levels are presented, the standard deviation of the mean (S.D.) and the number $(n)$ of independent experiments is indicated in each case. A probability value of $<0.05$ was considered statistically significant.

Acknowledgements. We appreciate the help of Dr. C Müller and Dr. E Binda regarding maintaining and genotyping of the mouse strains. We also thank Dr. D Simon (Department of Dermatology, Inselspital, University of Bern), who collected blood samples from control individuals. This work was supported by the Swiss National Science Foundation (Grant no. 310000-107526) and the OPO Foundation, Zurich, Switzerland.

1. Simon HU. Neutrophil apoptosis pathways and their modifications in inflammation. Immunol Rev 2003; 193: 101-110.

2. Lum JJ, Bren G, McClure R, Badley AD. Elimination of senescent neutrophils by TNF-related apoptosis-inducing ligand. J Immunol 2005; 175: 1232-1238.

3. Dibbert B, Weber M, Nikolaizik WH, Vogt P, Schöni MH, Blaser K et al. Cytokine-mediated Bax deficiency and consequent delayed neutrophil apoptosis: a general mechanism to accumulate effector cells in inflammation. Proc Natl Acad Sci USA 1999; 96: 13330-13335.

4. Saba S, Soong G, Greenberg S, Prince A. Bacterial stimulation of epithelial G-CSF and GM-CSF expression promotes PMN survival in CF airways. Am J Respir Cell Mol Biol 2002; 27: 561-567.

5. Jonsson H, Allen P, Peng SL. Inflammatory arthritis requires Foxo3a to prevent Fas ligandinduced neutrophil apoptosis. Nat Med 2005; 11: 666-671.

6. Conus S, Perozzo R, Reinheckel T, Peters C, Scapozza L, Yousefi S et al. Caspase-8 is activated by cathepsin $D$ initiating neutrophil apoptosis during the resolution of inflammation. J Exp Med 2008; 205: 685-698.

7. Hallett JM, Leitch AE, Riley NA, Duffin R, Haslett C, Rossi AG. Novel pharmacological strategies for driving inflammatory cell apoptosis and enhancing the resolution of inflammation. Trends Pharmacol Sci 2008; 29: 250-257.

8. Youle RJ, Strasser A. The Bcl-2 protein family: opposing activities that mediate cell death. Nat Rev Mol Cell Biol 2008; 9: 47-59.

9. Weinmann $P$, Gaehtgens $P$, Walzog B. Bcl- $x_{L}$ and Bax- $\alpha$-mediated regulation of apoptosis of human neutrophils via caspase-3. Blood 1999; 93: 3106-3115.

10. Moulding DA, Akgul C, Derouet M, White MR, Edwards SW. Bcl-2 family expression in human neutrophils during delayed and accelerated apoptosis. J Leukoc Biol 2001; 70: 783-792.

11. Moulding DA, Quayle JA, Hart CA, Edwards SW. Mcl-1 expression in human neutrophils: regulation by cytokines and correlation with cell survival. Blood 1998; 92: 2495-2502.

12. Epling-Burnette PK, Zhong B, Bai F, Jiang K, Bailey RD, Garcia R et al. Cooperative regulation of $\mathrm{Mcl}-1$ by Janus kinase/STAT and phosphatidylinositol 3-kinase contribute to granulocyte-macrophage colony-stimulating factor-delayed apoptosis in human neutrophils. J Immunol 2001; 166: 7486-7495.

13. Chuang PI, Yee E, Karsan A, Winn RK, Harlan JM. A1 is a constitutive and inducible Bcl-2 homologue in mature human neutrophils. Biochem Biophys Res Commun 1998; 249: 361-365.

14. Villunger A, O'Reilly LA, Holler N, Adams J, Strasser A. Fas ligand, Bcl-2, granulocyte colony-stimulating factor, and p38 mitogen-activated protein kinase: Regulators of distinct cell death and survival pathways in granulocytes. J Exp Med 2000; 192: 647-658.

15. Iwai K, Miyawaki T, Takizawa T, Konno A, Ohta K, Yachie A et al. Differential expression of Bcl-2 and susceptibility to anti-Fas-mediated cell death in peripheral blood lymphocytes, monocytes, and neutrophils. Blood 1994; 84: 1201-1208.

16. Bouillet $P$, Metcalf $D$, Huang DC, Tarlinton DM, Kay TW, Köntgen F et al. Proapoptotic $\mathrm{Bcl}-2$ relative Bim required for certain apoptotic responses, leukocyte homeostasis, and to preclude autoimmunity. Science 1999; 286: 1735-1738. 
17. Dijkers PF, Medema RH, Lammers JW, Koenderman L, Coffer PJ. Expression of the pro-apoptotic Bcl-2 family member Bim is regulated by the forkhead transcription factor FKHR-L1. Curr Biol 2000; 10: 1201-1204.

18. Whitfield J, Neame SJ, Paquet L, Bernard O, Ham J. Dominant-negative c-Jun promotes neuronal survival by reducing Bim expression and inhibiting mitochondrial cytochrome release. Neuron 2001; 29: 629-643.

19. Putcha GV, Moulder KL, Golden JP, Bouillet P, Adams JA, Strasser A et al. Induction of $\mathrm{Bim}$, a proapoptotic BH3-only Bcl-2 family member, is critical for neuronal apoptosis. Neuron 2001; 29: 615-628.

20. Akiyama T, Bouillet P, Miyazaki T, Kadono Y, Chikuda H, Chung UI et al. Regulation of osteoclast apoptosis by ubiquitylation of proapoptotic $\mathrm{BH} 3-$ only $\mathrm{Bcl}-2$ family member Bim. EMBO J 2003; 22: 6653-6664.

21. Ley R, Ewings KE, Hadfield K, Cook SJ. Regulatory phosphorylation of Bim: sorting out the ERK from the JNK. Cell Death Differ 2005; 12: 1008-1014.

22. Villunger $A, S$ cott $C$, Bouillet $P$, Strasser $A$. Essential role for the BH3-only protein Bim but redundant roles for $\mathrm{Bax}, \mathrm{Bcl}-2$, and $\mathrm{Bcl}-\mathrm{w}$ in the control of granulocyte survival. Blood 2003 101: 2393-2400.

23. O'Reilly LA, Cullen L, Visvader J, Lindeman GJ, Print C, Bath ML et al. The proapoptotic $\mathrm{BH} 3-$ only protein Bim is expressed in hematopoietic, epithelial, neuronal, and germ cells. Am J Pathol 2000; 157: 449-461.

24. Puthalakath H, Huang DC, O'Reilly LA, King SM, Strasser A. The proapoptotic activity of the Bcl-2 family member Bim is regulated by interaction with the dynein motor complex. Mol Cell 1999; 3: 287-296.

25. Day CL, Puthalakath H, Skea G, Strasser A, Barsukov I, Lian LY et al. Localization of dynein light chains 1 and 2 and their pro-apoptotic ligands. Biochem J 2004; 377: 597-605.

26. Altznauer F, Martinelli S, Yousefi S, Thürig C, Schmid I, Conway EM et al. Inflammationassociated cell cycle-independent block of apoptosis by survivin in terminally differentiated neutrophils. J Exp Med 2004; 199: 1343-1354.

27. Martinelli S, Urosevic M, Daryadel A, Oberholzer PA, Baumann C, Fey MF et al. Induction of genes mediating interferon-dependent extracellular trap formation during neutrophil differentiation. J Biol Chem 2004; 279: 44123-44132.

28. Chen M, Huang L, Wang J. Deficiency of Bim in dendritic cells contributes to overactivation of lymphocytes and autoimmunity. Blood 2007; 109: 4360-4367.

29. Dijkers PF, Birkenkamp KU, Lam EW, Thomas NS, Lammers JW, Koenderman L et al. FKHR-L1 can act as a critical effector of cell death induced by cytokine withdrawal: protein kinase B-enhanced cell survival through maintenance of mitochondrial integrity. J Cell Biol 2002; 156: 531-542

30. Stahl M, Dijkers PF, Kops GJ, Lens SM, Coffer PJ, Burgering BM et al. The forkhead transcription factor Foxo regulates transcription of p27Kip1 and Bim in response to IL-2. $J$ Immunol 2002; 168: 5024-5031.

31. Gilley J, Coffer PJ, Ham J. Foxo transcription factors directly activate Bim gene expression and promote apoptosis in sympathetic neurons. J Cell Biol 2003; 162: 613-622.

32. Jönsson $M$, Engström M, Jönsson Jl. FLT3 ligand regulates apoptosis through AKT-dependent inactivation of transcription factor Fox03. Biochem Biophys Res Commun 2004; 318: 899-903.

33. Möller C, Alfredsson J, Engström M, Wootz H, Xiang Z, Lennartsson J et al. Stem cell factor promotes mast cell survival via inactivation of Foxo3a-mediated transcriptional induction and MEK-regulated phosphorylation of the proapoptotic protein Bim. Blood 2005; 106 : 1330-1336.

34. Rosas M, Dijkers PF, Lindemans CL, Lammers JW, Koenderman L, Coffer PJ. IL-5mediated eosinophil survival requires inhibition of GSK-3 and correlates with $\beta$-catenin relocalization. J Leukoc Biol 2006; 80: 186-195.

35. Qi XJ, Wildey GM, Howe PH. Evidence that Ser87 of Bim $\mathrm{EL}_{\mathrm{L}}$ is phosphorylated by Akt and regulates Bim ${ }_{E L}$ apoptotic function. J Biol Chem 2006; 281: 813-823.

36. Kirschnek S, Ying S, Fischer SF, Häcker H, Villunger A, Hochrein $\mathrm{H}$ et al. Phagocytosisinduced apoptosis in macrophages is mediated by up-regulation and activation of the Bcl-2 homology domain 3-only protein Bim. J Immunol 2005; 174: 671-679.

37. Alfredsson J, Puthalakath H, Martin H, Strasser A, Nilsson G. Proapoptotic Bcl-2 family member Bim is involved in the control of mast cell survival and is induced together with $\mathrm{Bcl}-\mathrm{x}_{\mathrm{L}}$ upon IgE-receptor activation. Cell Death Differ 2005; 12: 136-144.

38. Bauer A, Kirschnek S, Häcker G. Inhibition of apoptosis can be accompanied by increased Bim levels in T lymphocytes and neutrophil granulocytes. Cell Death Differ 2007; 14: 1714-1716

39. Zhu Y, Swanson BJ, Wang M, Hildeman DA, Schaefer BC, Liu X et al. Constitutive association of the proapoptotic protein Bim with Bcl-2-related proteins on mitochondria in $\mathrm{T}$ cells. Proc Natl Acad Sci USA 2004; 101: 7681-7686.

40. Gomez-Bougie $\mathrm{P}$, Bataille R, Amiot $\mathrm{M}$. Endogenous association of Bim BH3-only protein with Mcl-1, Bcl- $x_{\mathrm{L}}$ and Bcl-2 on mitochondria in human B cells. Eur J Immunol 2005; 35: 971-976.

41. Downey GP, Butler JR, Tapper H, Fialkow L, Saltiel AR, Rubin BB et al. Importance of MEK in neutrophil microbicidal responsiveness. J Immunol 1998; 160: 434-443.

42. Dimitriou ID, Clemenza L, Scotter AJ, Chen G, Guerra FM, Rottapel R. Putting out the fire: coordinated suppression of the innate and adaptive immune systems by SOCS1 and SOCS3 proteins. Immunol Rev 2008; 224: 265-283.

43. Martinez-Moczygemba M, Huston DP. Proteasome regulation of $\beta \mathrm{c}$ signaling reveals a novel mechanism for heterotypic desensitization. J Clin Invest 2001; 108: 1797-1806.

44. Levy BD, Clish CB, Schmidt B, Gronert K, Serhan CN. Lipid mediator class switching during acute inflammation: signals in resolution. Nat Immunol 2001; 2: 612-619.

45. Bellocchio S, Moretti S, Perruccio K, Fallarino F, Bozza S, Montagnoli C et al. TLRs govern neutrophil activity in aspergillosis. J Immunol 2004; 173: 7406-7415.

46. Kostylina G, Simon D, Fey MF, Yousefi S, Simon HU. Neutrophil apoptosis mediated by nicotinic acid receptors (GPR109A). Cell Death Differ 2008; 15: 134-142.

47. Martinelli S, Kostylina G, Niggli V, Baumann C, Fey MF, Wendel HG et al. Targeting survivin via $\mathrm{PI} 3 \mathrm{~K}$ but not $\mathrm{c}-\mathrm{akt} / \mathrm{PKB}$ by anticancer drugs in immature neutrophils. Oncogene 2006; 25: 6915-6923

48. Vassina EM, Yousefi S, Simon D, Zwicky C, Conus S, Simon HU. clAP-2 and survivin contribute to cytokine-mediated delayed eosinophil apoptosis. Eur J Immunol 2006; 36: 1975-1984.

49. Yousefi S, Gold JA, Andina N, Lee JJ, Kelly AM, Kozlowski E et al. Catapult-like release of mitochondrial DNA by eosinophils contributes to antibacterial defense. Nat Med 2008; 14: 949-953.

50. Kaufmann $\mathrm{T}$, Jost PJ, Pellegrini M, Puthalakath $\mathrm{H}$, Gugasyan $\mathrm{R}$, Gerondakis $\mathrm{S}$ et al. Fatal hepatitis mediated by tumor necrosis factor TNF $\alpha$ requires caspase- 8 and involves the BH3-only proteins Bid and Bim. Immunity 2009; 30: 56-66. 